\title{
Adherence to European Polypectomy Guidelines: Retrospective Experience from a Tertiary Irish Hospital
}

\author{
Vikrant Parihara Julia Sopheno-Falco $^{a}$ Pardeep Maheshwaria Neil O'Moran ${ }^{a}$ \\ Vivien Graziadei $^{a} \quad$ Aishling O'Grady Walshe ${ }^{a}$ Orla O'Dwyer ${ }^{a} \quad$ Lakshman Kumar $^{a}$ \\ Sean Fennessy ${ }^{a}$ Niall Breslina Barbara M. Ryan ${ }^{a}$ Deirdre McNamara ${ }^{a-c}$ \\ ${ }^{a}$ Department of Gastroenterology AMNCH, Dublin, Ireland; ${ }^{b}$ Trinity College Dublin, Dublin, Ireland; \\ 'TAGG (Trinity Academic Gastroenterology Group), Dublin, Ireland
}

\section{Keywords}

Polypectomy · Adherence rates $\cdot$ Snares

\begin{abstract}
Background and Study Aim: The European guidelines for colorectal cancer screening state that snare resection should remove any polyps $\geq 5 \mathrm{~mm}$. This study aimed to investigate if these new guidelines are adhered to in clinical practice. Patients and Methods: This study consists of patients who underwent colonoscopies in Tallaght Hospital, Dublin (AMNCH), between 2012 and 2015. The size of the polyp, the method of removal, and the subspecialty and grade of the endoscopists were all recorded. Results: 6,000 colonoscopies were reviewed and 687 (12.5\%) of these patients were found to have polyps. In 655 (95\%) colonoscopies, the caecum was positively identified. In all, $371(54 \%)$ of the polyps detected were $<5 \mathrm{~mm}$; resection via forceps was carried out in $n 405$ cases (59\%). Overall, 16\% $(n=45)$ of the polyps $>5 \mathrm{~mm}$ underwent resection with forceps, showing that the new European guidelines are not being tightly adhered to. Conclusions: This study found an $84 \%$ compliance with polypectomy resection guidelines which is an improvement on previous studies. However, endoscopist grade significantly affected compliance and may reflect overall competency, highlighting the need for specific training in snare polypectomy techniques.

(C) 2018 S. Karger AG, Base
\end{abstract}

\section{Introduction}

In Ireland, colorectal cancer (CRC) made up 11\% of all cancers in women and $14 \%$ of men in 2007-2009, making it the second-most common cancer in both sexes. It was the third leading cause of cancer death in women and the second in men in this period. Between 1994 
and 2010, the number of CRC cases increased by $2.1 \%$ annually [1]. The adenoma (as well as the serrated adenoma sequence of colorectal carcinogenesis) lends itself to screening and surveillance, albeit at differing intervals [2]. Studies have shown that the complete removal of colonic polyps during colonoscopy reduces the incidence of CRC [3]. It has also been estimated that incomplete resection is involved in 19-31\% of interval cancers [3]. Studies have shown that, $10-15 \%$ of the time, residual polypoid tissue can be found at the post-polypectomy site $[4,5]$. This means that, along with correct screening and detection techniques, the method used for resecting of polyps is becoming increasingly important. The two most common methods of removal of small sessile polyps are via biopsy forceps and snare.

The new European guidelines for quality assurance in CRC screening and diagnosis state that: "Colorectal lesions should only be removed by endoscopists with adequate training in techniques of polypectomy. Lesions $>5 \mathrm{~mm}$ must be removed by snaring while those $<5 \mathrm{~mm}$ may be removed with biopsy forceps" [6]. This guideline highlights the need for adequate regulations regarding polyp resection. A study by Park et al. [7] showed that removal of polyps 1-3 mm in size by cold forceps had the same outcome as those removed by cold-snare resection, but that cold-forceps polypectomy is likely to be insufficient for larger polyps. On the other hand, cold-snare techniques reduce the risk of incomplete diminutive polyp removal by as much as $60 \%$, without increasing the total procedure time [8]. Snare polypectomy also has the advantage of being able to remove the polyp as a whole, rather than in the piecemeal fashion associated with cold biopsy, making it easier for the pathologist to ascertain complete histological removal. To improve patient outcomes, it is important to not only disseminate this knowledge but to promote adherence in clinical practice. We aimed to verify if the new guidelines are in fact being adhered to locally.

\section{Aim}

To investigate polypectomy techniques, adherence to guidelines, and differences between subspecialty and consultants versus trainees in a real-world colonoscopy cohort, as part of an institutional quality improvement audit.

\section{Methods}

\section{Study Participation}

This was a retrospective non-randomised observational study on a colonoscopy cohort between January 2012 and December 2015. All these procedures were conducted in a single tertiary hospital, Tallaght Hospital, Ireland. All patients who underwent colonoscopy regardless of the indication in Tallaght Hospital during the above period were eligible for the study.

\section{Exclusion Criteria}

Exclusion criteria included: incomplete colonoscopy, inadequate bowel preparation, contra-indication to polypectomy including coagulopathy, severe inter-current illness, repeat interval procedures for followup of recent endoscopic mucosal resection or large polypectomy, previous colectomy, surgery, assessment of active inflammation, and known CRC.

\section{Study Outcome}

A list of patients with colonic polyps was compiled. Only the largest or most histologically advanced polyp was analysed when examining the method of removal and retrieval. Demographics and other information including the number and site of the polyp, resection and retrieval rates, resection methods, histology, and the specialty and grade of the endoscopist were recorded. Polyp location was recorded, with polyps from the caecum to the splenic flexure regarded as being on the right, and those from the splenic flexure to the rectum as being on the left. Polyp size was based on the pathologist's assessment. As it was a retrospective 
Parihar et al.: Adherence to European Polypectomy Guidelines: Retrospective Experience from a Tertiary Irish Hospital

Fig. 1. Indications for colonoscopy. IBD, inflammatory bowel disease; CRC, colorectal cancer.

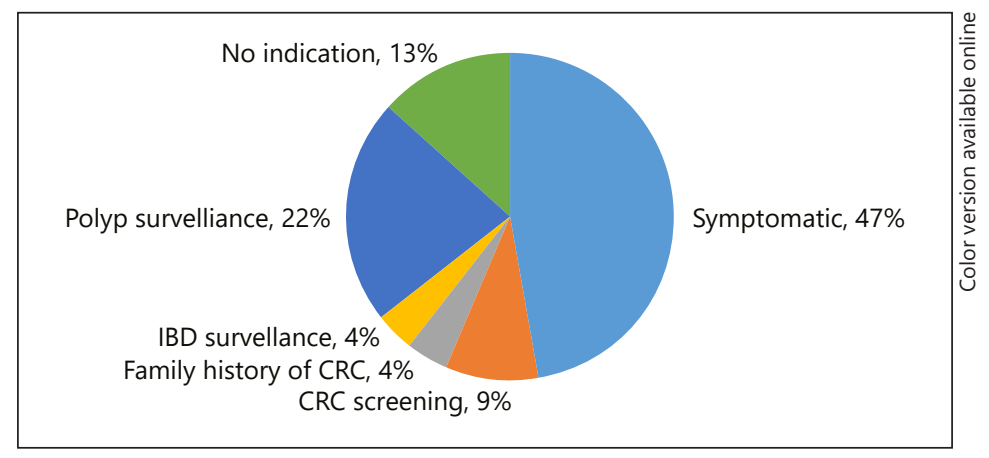

review with data on patients retrieved without any new intervention, the approval of an ethics committee was not deemed necessary. SPSS software was used for statistical analysis. Statistical significance was set a priori at $p<0.05$

\section{Results}

\section{Patients}

A total of 11,400 colonoscopies were performed during the period of the study. To date, records of around $6,000(53 \%$ of the 11,400) consecutive procedures have been reviewed; $504(8.4 \%)$ colonoscopies were excluded from the study as per the exclusion criteria specified above, and $687(12.5 \%)$ colonoscopies with polyps were analysed.

This included 298 female (43\%) and 389 male (57\%) patients with a mean age of 60.62 years (SD 12.38). The colonoscopies were performed in symptomatic patients in $47 \%$. The rest were for polyp surveillance (22\%), CRC screening (9\%), a family history of CRC (4\%), and inflammatory bowel disease (IBD) surveillance (4\%) (Fig. 1). For 13\% of the included patients, no indication for colonoscopy was recorded.

The caecal intubation rate was $95.3 \%(n=655)$. The caecum was not reached in $3.9 \%$ of cases $(n=27)$ and no information was supplied about caecal intubation in 5 cases $(0.7 \%)$. Failure to intubate the caecum occurred in $4 \%(n=7)$ of the colonoscopies carried out by medical consultants, $3 \%(n=11)$ carried out by medical registrars, $2 \%(n=1)$ performed by surgical consultants, and 7\% $(n=8)$ undertaken by surgical registrars; there was no statistically significant difference across these grades. The mean number of polyps per patient was 2.2 (SD 1.889), with $48 \%$ of patients having just 1 polyp identified. The maximum number of polyps observed in 1 patient was 15 . In $52.5 \%(n=361)$ of the patients, polyps were recorded as being on the left, and they were on the right in $23.1 \%(n=159)$. Polyps were found on both sides in $23.7 \%$ of cases ( $n=159)$, and no location of the resected polyps was given for $0.5 \%$ of the patients $(n=4)$. In all $316(46 \%)$ patients, the polyps were $>5 \mathrm{~mm}$ in diameter.

\section{Forceps and Snare Usage by Lesion Size}

In total, 59\% ( $n=405)$ of lesions were resected using cold forceps, 5.37\% ( $n=36)$ using hot forceps, $17 \%(n=116)$ using a cold snare, and 19\% $(n=130)$ using a hot snare. Of the resected index polyps, $54 \%(n=363)$ were $<5 \mathrm{~mm}, 36.33 \%(n=246) 5-10 \mathrm{~mm}$, and $10.04 \%$ $(n=68)>10 \mathrm{~mm}$.

Of the 314 polyps $\geq 5 \mathrm{~mm}, 108$ (accounting for $16 \%$ of all procedures studied) were resected inappropriately using forceps ( 97 with cold forceps and 11 with hot forceps). The largest polyp removed using forceps was $15 \mathrm{~mm}$ in size. Table 1 displays these procedures broken down by the level of training of the endoscopist. Medical registrars performed $54 \%$ 


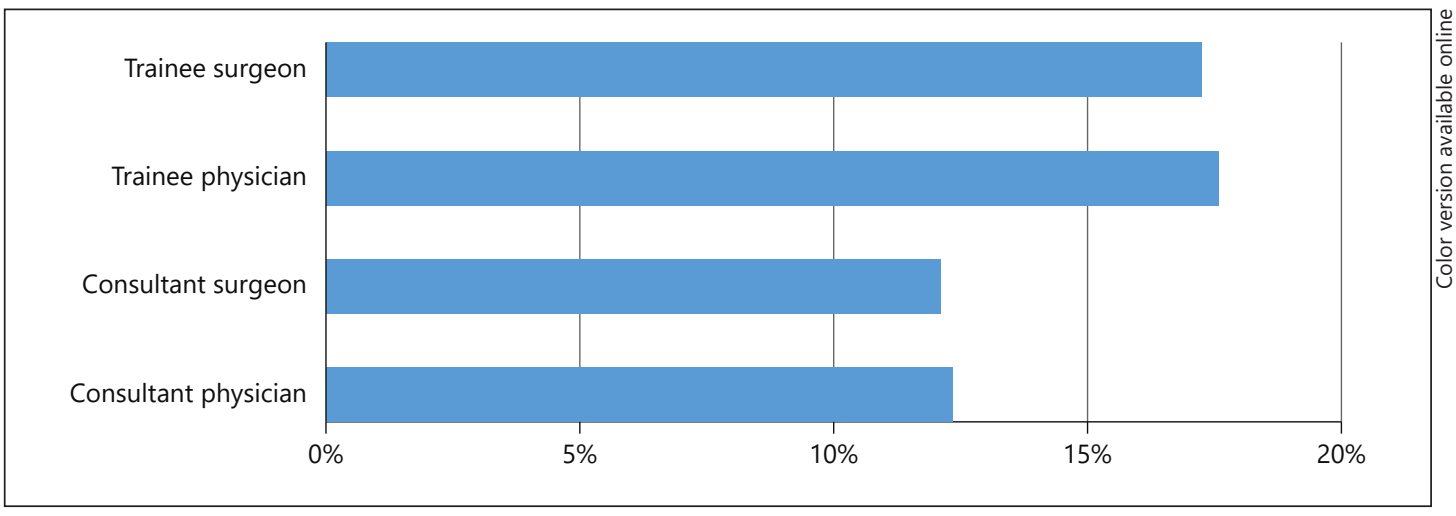

Fig. 2. Non-adherence rates.

Table 1. Procedures not in compliance with the guidelines

\begin{tabular}{lc}
\hline Endoscopist grade & Polypectomies/noncompliance, $N / n$ \\
\hline Medical consultant & $195 / 24$ \\
Medical registrar & $330 / 58$ \\
Surgical consultant & $41 / 5$ \\
Surgical registrar & $121 / 21$ \\
\hline
\end{tabular}

of polypectomies that did not adhere to guidelines, and medical consultants carried out $22 \%$ of the procedures. Surgical registrars carried out 20\%, with surgical consultants performing $4.64 \%$ of the non-compliant polypectomies. Polypectomies which did not adhere to guidelines accounted for $18 \%$ of all polypectomies performed by medical registrars, $12 \%$ of those carried out by medical consultants, $12 \%$ of those carried out by surgical consultants, and $17 \%$ of those carried out by surgical registrars (Fig. 2).

There was no difference in the size of the polyps removed among specialties and grades but medical trainees versus consultants nearly achieved statistical significance in polypectomy method compliance by using snares to tackle polyps $\geq 5 \mathrm{~mm}(p<0.075$ and 0.062$)$. Of note, $59 \%(n=143)$ of the polyps that were $5-10 \mathrm{~mm}$ in size were removed as per the guidelines, compared to $85 \%(n=58)$ of those $>10 \mathrm{~cm}$, suggesting that endoscopists were 4 times more likely to snare bigger lesions (OR 4.01, 95\% CI 1.957-8.23, $p<0.00019$ ).

\section{Endoscopists and Polyp Retrieval}

Medical endoscopists, i.e., consultants and trainees, performed more than three quarters of the procedures (195 [28\%] and 330 [48\%], respectively) with 41 surgical consultants (6\%) and 121 trainees $(18 \%)$ performing the rest. The polypectomies carried out by each subgroup of endoscopists that did not conform to the guidelines as per the specialty and grade were: 24 medical consultants (12.3\%), 58 medical trainees (17.57\%), 5 surgical consultants (12.1\%), and 21 surgical trainees (17.35\%) (Table 1; Fig. 2). While these findings did not reach statistical significance, trainee endoscopists were 1.5 times more likely to remove polyps $>5 \mathrm{~mm}$ by the cold-forceps technique (OR 1.51, 95\% CI $0.95-2.39, p<0.07$ ). Of all resected polyps, only $1.75 \%$ were not retrieved $(n=12)$. Medical consultants were the lead endoscopists in $3(1.6 \%)$ of these cases, medical registrars in $4(1.2 \%)$, and surgical registrars in $5(4 \%)$. 


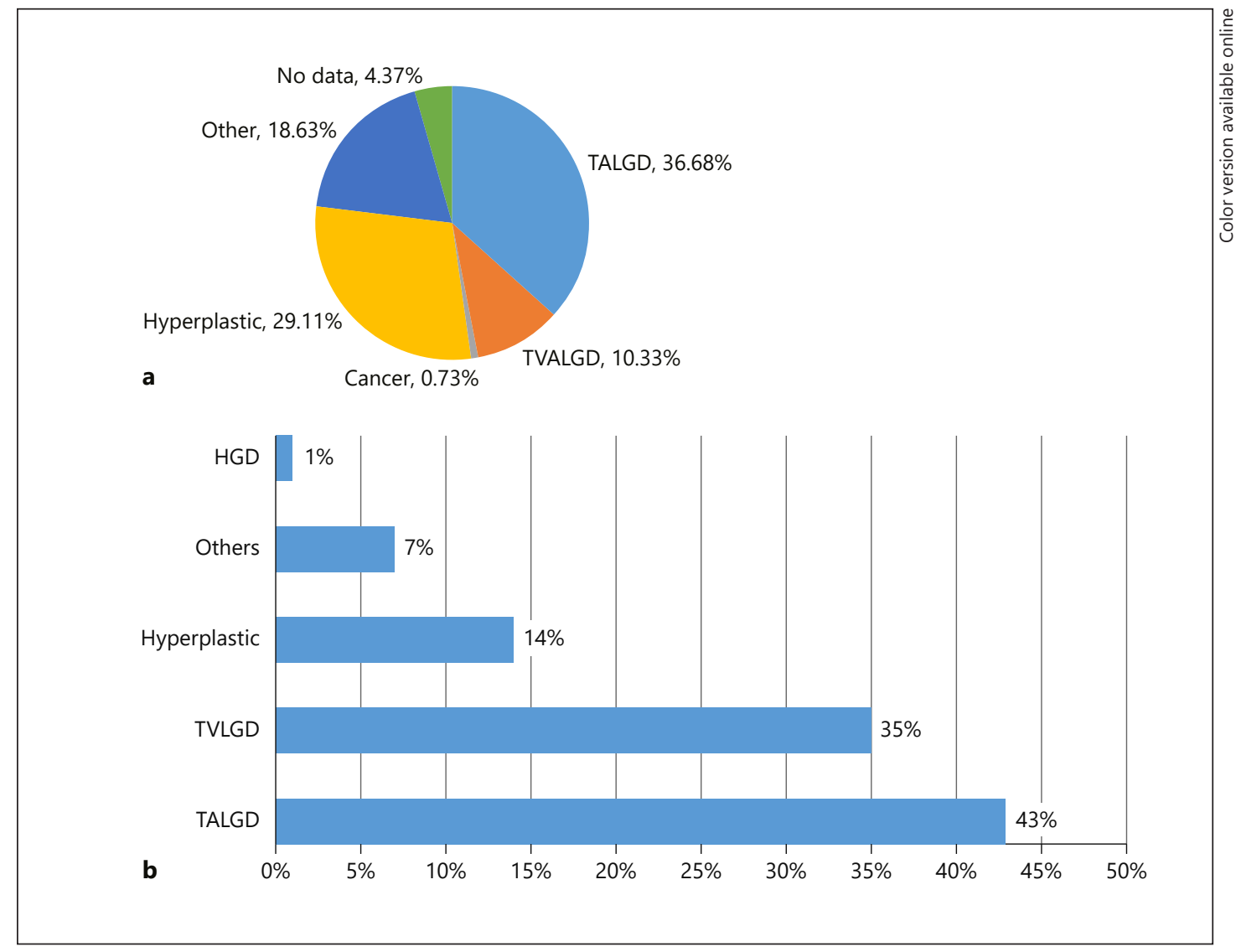

Fig. 3. a Histology of resected polyps. b Histology of inappropriately removed polyps. TALGD, tubular adenoma with low-grade dysplasia; TVALGD, tubulovillous adenoma with low-grade dysplasia; HGD, high-grade dysplasia.

\section{Polyp Histology}

The most common polyp histology was tubular adenoma with low-grade dysplasia (37\%) followed by hyperplastic lesions (29\%), others (19\%), and tubulovillous adenoma with lowgrade dysplasia (10\%). Advanced histology occurred in 4.73\%: high-grade dysplasia (4\%) and cancer $(0.73 \%)$. The histology of inappropriately resected polyps was: 84 adenomas (78\%), 25 hyperplastic (23\%) and 1 high-grade dysplasia (1\%), and 8 others (7.5\%). More importantly, a disproportionately higher number of adenomas were inappropriately removed (78\%) (Fig. 3). In addition, polyps $>5 \mathrm{~mm}$ were statistically more likely to be tubulovillous adenomas.

\section{Discussion}

In this retrospective study, 687 colonoscopies with polyps from a university-affiliated training unit at Tallaght Hospital, performed between January 2012 and December 2015, were analysed. In this large single-center study with overall acceptable colonoscopy standards based on current quality indicators, one of the principal aims was to determine endoscopist adherence to current polypectomy guidelines, which recommend that polyps $\geq 5 \mathrm{~mm}$ be removed using a snare. 
This is very important as it has been shown that removing small sessile colonic polyps identified during colonoscopy reduces the incidence of CRC [9]. Recent studies by Park et al. [7] and Kim et al. [10] also showed that the cold-snare technique consistently achieved higher complete resection rates for polyps 5-7 $\mathrm{mm}$ in size than cold forceps. This finding agrees with that of Urquhart and Brown [9] who found that a cold snare can be consistent in achieving a complete resection of sessile polyps when a 1- to 2-mm rim of normal mucosa around the polyp is also resected.

Our study found that $15.72 \%$ of the polypectomies analysed did not adhere to the guidelines, meaning that the presence of residual abnormal tissue cannot be ruled out. This is comparable to prior studies [4]. On average, consultants were not compliant in $12.26 \%$ of their cases, and trainees were not compliant in $17.47 \%$ of theirs. This is significant, as $48 \%$ $(n=330)$ of all polypectomies studied were performed by medical trainees and $17.6 \%(n=$ 121) were carried out by surgical trainees. This highlights the need for an enhanced awareness of appropriate techniques and the benefits of snaring these lesions. As we did not ask the endoscopists if they knew of the European Society of Gastrointestinal Endoscopy (ESGE) recommendation or were aware of the risks factors for incomplete resection, our findings could simply reflect a lack of awareness. This is the most likely explanation as we found compliance with other colonoscopy quality indicators and training oversight.

Several studies have indicated that there is a considerable rate of residual polyp tissue after what was thought to be a complete polypectomy by cold forceps $[4,10]$. Studies prior to the issuing of the guidelines show a significantly higher rate of inappropriate forceps use, sometimes as high as $46 \%$; studies show a decrease of $14.2 \%$ in inappropriate forceps use in hospitals since the new guidelines came into place [3]. This is in keeping with our data. We recorded the rate of caecal intubation as an indicator of the quality of the colonoscopies carried out. It has been found that effective colonoscopists should succeed in caecum intubation in $\geq 90 \%$ of all cases and screening in 95\% [11]. Reassuringly, endoscopists intubated the caecum in $95.3 \%(n=655)$ of the colonoscopies analysed in this study. We found little correlation between the level of training or specialty and rate of caecal intubation, with medical and surgical consultants and medical trainees succeeding in $>96 \%$ of cases $(n=547)$, and surgical registrars succeeding in $93.39 \%(n=113)$. This can be explained by the fact that Tallaght Hospital is a teaching university hospital and a trainer is available to help trainees.

Overall, the polyp detection rate in this study was low (around 13\%). This is probably related to our cohort as most of the colonoscopies were done on symptomatic patients and not for screening. However, unlike other studies [12] no difference in this rate was found across grade and specialty, again likely reflecting the indication for colonoscopy in an acute hospital setting and the availability of trainers in a teaching facility.

There were several limitations which should be noted in this study. Regarding the method of removal and retrieval, only the index polyp was analysed. This could have limited the insight that can be gained from the study. However, we felt it was reasonable to exclude polyps $<5 \mathrm{~mm}$ from the analysis as these were not under consideration as part of the guidelines and most of our cohort had only 1 polyp excised. In addition, if the largest polyp (index lesion) was not removed appropriately, our data is likely to have under-represented the problem and suggests that the findings do accurately reflect real-world practice. Since the study was conducted, there has been an ESGE recommendation of cold-snare polypectomy as the preferred technique for the removal of diminutive polyps $(\leq 5 \mathrm{~mm})$. This technique has high rates of complete resection, adequate tissue sampling for histology, and low complication rates [13].

Furthermore, endoscopists did not record the lesion size in 30\% of cases. This, in itself, is a significant finding and warrants future action to improve accurate reporting. Documenting lesion size is an essential aspect of colonoscopy which can enable the appropriate 
interpretation of findings, and determine the quality of the procedure as well as being used for scheduling appropriate follow-up. The data provided on colonoscopy reports was recorded by individual colonoscopists themselves and, as such, there is a further chance of inaccuracy. However, this is the norm in clinical practice. Since we only analysed the index polyp, we were unable to report our polyp retrieval rate, another key performance indicator. To overcome these issues, we used the pathologist assessment of size, which, in the case of piecemeal resection, was an estimate.

Unfortunately, the design of our study means there is no data available on the actual rate of residual polyp tissue overall or based on the excision technique. It was not possible to check if there was any residual lesion in the polypectomies performed using forceps. This was not the aim of our study, however, and we assume from the available data that non-compliance with appropriate polypectomy technique does increase the risk of incomplete resection. Further assessment of pathological resection is part of an ongoing prospective study in our centre.

\section{Conclusions}

We found that between January 2012 and December 2015, contrary to what is stipulated in the European guidelines for quality assurance, $16 \%$ of the polyps $\geq 5 \mathrm{~mm}$ in size were resected using forceps. This is lower than levels found in recent similar studies in other countries. Trainee endoscopists were 1.5 times more likely to use inappropriate resection techniques, and we would recommend that additional focused training on polypectomy technique would be beneficial. In addition, polypectomy technique could and should be an independent quality indicator for colonoscopy. Finally, there is the need for an awareness campaign, as simply publishing a guideline, while it has some impact, does not completely address the knowledge gap.

\section{Disclosure Statement}

None of the authors has any financial or conflict of interest disclosures.

\section{References}

1 Colorectal Cancer Incidence, Mortality, Treatment and Survival in Ireland: 1994-2010. National Cancer Registry, Ireland.

2 Vogelstein B, Papadopoulos N, Velculescu VE, Zhou S, Diaz LA Jr, Kinzler KW. Cancer genome landscapes. Science. 2013 Mar;339(6127):1546-58.

3 Britto-Arias M, Waldmann E, Jeschek P, Gessl I, Sallinger D, Bannert C, et al. Forceps versus snare polypectomies in colorectal cancer screening: are we adhering to the guidelines? Endoscopy. 2015 Oct;47(10):898902.

4 Liu S, Ho SB, Krinsky ML. Quality of polyp resection during colonoscopy: are we achieving polyp clearance? Dig Dis Sci. 2012 Jul;57(7):1786-91.

5 Pohl H, Srivastava A, Bensen SP, Anderson P, Rothstein RI, Gordon SR, et al. Incomplete polyp resection during colonoscopy-results of the complete adenoma resection (CARE) study. Gastroenterology. 2013 Jan;144(1): 74-80.e1.

6 Steele RJ, Pox C, Kuipers EJ, Minoli G. European guidelines for quality assurance in colorectal cancer screening and diagnosis. 1st edition - Management of lesions detected in colorectal cancer screening. Endoscopy. 2012; 44:SE140-SE150.

7 Park SK, Ko BM, Han JP, Hong SJ, Lee MS. A prospective randomized comparative study of cold forceps polypectomy by using narrow-band imaging endoscopy versus cold snare polypectomy in patients with diminutive colorectal polyps. Gastrointest Endosc. 2016 Mar;83(3):527-32.e1. 
8 Raad D, Tripathi P, Cooper G, Falck-Ytter Y. Role of the cold biopsy technique in diminutive and small colonic polyp removal: a systematic review and meta-analysis. Gastrointest Endosc. 2016 Mar;83(3):508-15.

9 Urquhart P, Brown G. The Effectiveness of Cold Snare Polypectomy for the Removal of Small Sessile Colonic Polyps. Gastrointest Endosc. 2012;75(4):AB328.

10 Kim JS, Lee BI, Choi H, Jun SY, Park ES, Park JM, et al. Cold snare polypectomy versus cold forceps polypectomy for diminutive and small colorectal polyps: a randomized controlled trial. Gastrointest Endosc. 2015 Mar; 81(3):741-7.

11 Marshall JB, Barthel JS. The frequency of total colonoscopy and terminal ileal intubation in the 1990s. Gastrointest Endosc. 1993 Jul-Aug;39(4):518-20.

12 Ferlitsch M, Moss A, Hassan C, Bhandari P, Dumonceau JM, Paspatis G, et al. Colorectal polypectomy and endoscopic mucosal resection (EMR): European Society of Gastrointestinal Endoscopy (ESGE) Clinical Guideline. Endoscopy. 2017 Mar;49(3):270-97.

13 Bretthauer M, Kaminski MF, Løberg M, Zauber AG, Regula J, Kuipers EJ, et al.; Nordic-European Initiative on Colorectal Cancer (NordICC) Study Group. Population-Based Colonoscopy Screening for Colorectal Cancer: A Randomized Clinical Trial. JAMA Intern Med. 2016 Jul;176(7):894-902. 